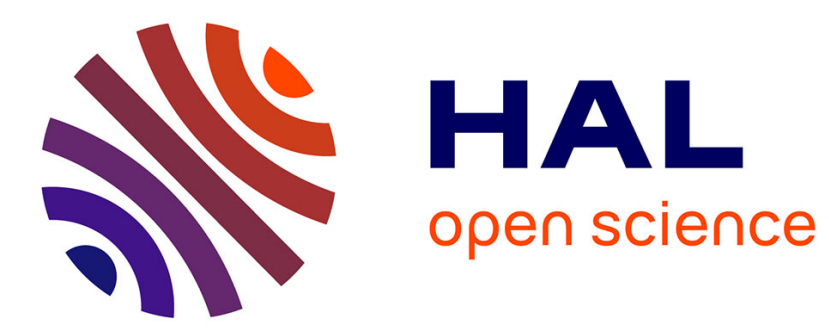

\title{
Realization of time-resolved two-vacuum-ultraviolet-photon ionization
}

A. Peralta Conde, J. Kruse, O. Faucher, P. Tzallas, E. P. Benis, D.

Charalambidis

\section{- To cite this version:}

A. Peralta Conde, J. Kruse, O. Faucher, P. Tzallas, E. P. Benis, et al.. Realization of time-resolved two-vacuum-ultraviolet-photon ionization. Physical Review A : Atomic, molecular, and optical physics [1990-2015], 2009, 79 (6), pp.061405(R). 10.1103/PhysRevA.79.061405 . hal-00402819

\section{HAL Id: hal-00402819 \\ https://hal.science/hal-00402819}

Submitted on 8 Jul 2009

HAL is a multi-disciplinary open access archive for the deposit and dissemination of scientific research documents, whether they are published or not. The documents may come from teaching and research institutions in France or abroad, or from public or private research centers.
L'archive ouverte pluridisciplinaire HAL, est destinée au dépôt et à la diffusion de documents scientifiques de niveau recherche, publiés ou non, émanant des établissements d'enseignement et de recherche français ou étrangers, des laboratoires publics ou privés. 


\title{
Realization of time resolved two-VUV-photon ionization
}

\author{
A. Peralta Conde ${ }^{1}$, J. Kruse ${ }^{1}$, O. Faucher ${ }^{2}$, P. Tzallas ${ }^{1}$, E.P. Benis ${ }^{1}$, and D. Charalambidis ${ }^{1,3}$ \\ 1 Foundation for Research and Technology Hellas, Institute of Electronic Structure and Laser, \\ P.O. Box 1385, GR71110 Heraklion (Crete), Greece. \\ 2 Institut Carnot de Bourgogne, UMR 5209 CNRS-Université de Bourgogne, \\ 9 Av. A. Savary, BP 47 870, F-21078 Dijon Cedex, France. \\ 3 Department of Physics, University of Crete, PO Box 2208, GR71003 Heraklion (Crete), Greece.
}

\begin{abstract}
Ultrafast dynamics of excited molecules is studied through time resolved two-VUV-photon ionization using a non-linear volume autocorrelator unit. The two-VUV-photon process is induced by the intense $5^{\text {th }}$ harmonic radiation of a fs Ti:Sapphire laser. In a proof-of-principle experiment, ultrafast dynamics of excited ethylene and oxygen molecules are investigated. Molecular decay times are deduced by comparing the experimental data with the results of a numerical model that accounts for the spatial and temporal characteristics of the harmonic field. The present experiments pave a convenient way for time domain investigations in the VUV/XUV spectral region in all states of matter at few fs to sub- fs temporal scales.
\end{abstract}

PACS numbers: 42.65.Re, 32.80.Rm, 42.65.Ky

Time resolved two vacuum ultraviolet (VUV) photon ionization measurements are initiated in the area of molecular dynamics, demonstrating their feasibility and strength in studying ultrafast phenomena. In nature many fundamental processes take place in femtosecond (fs) or even attosecond (asec) time scales. Real time investigations of such processes require ultrahigh temporal resolution. Most of the materials strongly absorb in the VUV (or extreme ultra-violet (XUV) spectral region) pointing the origin of the term "vacuum ultraviolet". Rapidly evolving quantum states may be excited through absorption of VUV radiation photons, while their dynamics may be probed by a second VUV photon absorbed at a later time. Thus, intense fs or sub-fs VUV/XUV pulses become an ideal instrument for recording snapshots of such ultrafast evolving systems. In the last decade, the substantial progress in the development of coherent XUV radiation sources of ultrashort pulse duration [1-4], together with the increased control over the laser pulse [5], have provided a powerful tool for the investigation of such ultrafast processes [6-8]. XUV-infrared (IR) cross correlation measurements underlie the majority of these measurements with the IR acting mainly as the time delayed probe. This was partially due to the lack of sufficient VUV/XUV intensities required for a pure VUV/XUVpump-VUV/XUV-probe measurement. However, the use of a hybrid IR/XUV interaction scheme, at often non negligible IR intensities, may introduce resonances with unwanted intermediate states, or involve the IR radiation in the pump process, and thus it may complicate the interpretation of the measured data. Experiments involving solely XUV-photons in both the excitation and the probing steps, would circumvent these complications. Recent campaigns in the field of intense XUV radiation sources, have reached intensities that allow for the observation of two-XUV-photon transitions even at sub-fs pulse durations [9-12], meeting the requirements for an XUV-pump-XUV-probe experiment.

In the present work, we report on the first study of ul- trafast molecular processes by means of the time resolved two-VUV-photon ionization technique. We will show how the dynamics of an excited electronic wavepacket of a molecule, decaying to the ground state via a sequence of quantum paths can be monitored, the different paths can be traced, and all the corresponding decay times may be deconvoluted from the measured time domain traces. The present results in conjunction with the upcoming new generation of intense asec pulses [13-15] establish a novel path for tracing ultrafast dynamics in atomic, molecular, macromolecular, and condensed matter systems.

Interferometric time resolved two-VUV-photon ionization reveals the dynamics of the driven wavepackets $[14,16]$. If the excitation is non-resonant the interferometric trace contains information about the temporal characteristics of the driven field $[14,16-18]$. If the excitation process is near resonant or resonant, the interferometric trace, even cycle-averaged, contains information about the temporal characteristics of the intermediate state. In principle, both pulse duration and characteristic time of the system can be deconvoluted from the measured trace. In practice, an independent measurement of the former quantity leads to more reliable results.

This new method is applied and tested in ethylene $\left(\mathrm{C}_{2} \mathrm{H}_{4}\right)$ and oxygen $\left(\mathrm{O}_{2}\right)$ molecules. Ethylene is the simplest molecule for studying the double $\mathrm{CC}$ bond. Although it has been investigated for a long time, there are still many open questions concerning its photochemical reaction dynamics in the UV/VUV spectral range [19$22]$. In the case of oxygen, the absorption of this molecule in the 7-9.8 eV UV/VUV region, what it is called the Schumann-Runge continuum, is of central importance to environmental issues.

The photoreaction path-network for ethylene that is considered in this work is shown in Fig. 1. It is similar to the one detailed in Ref. [22], and compatible with other previous works. The ethylene molecule is excited by the $5^{\text {th }}$ harmonic of a Titanium-Sapphire femtosecond laser 


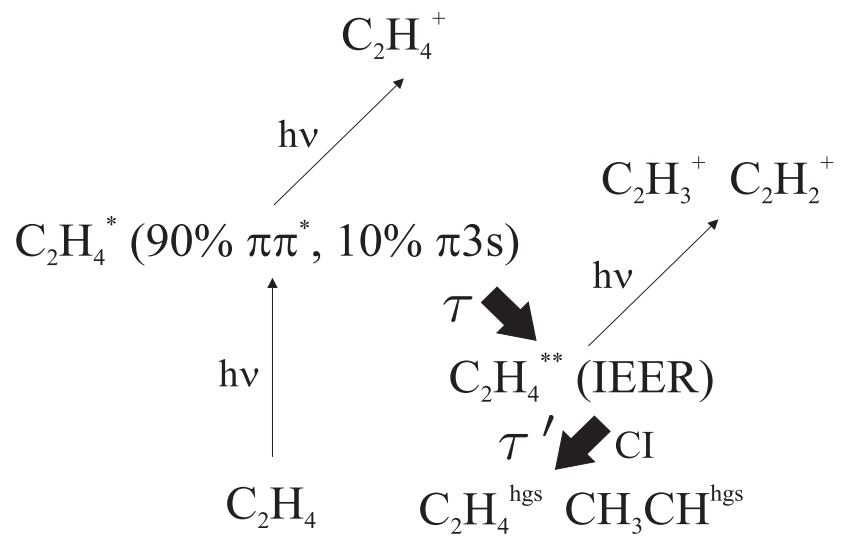

FIG. 1: Photoreaction path-network. IEER: intermediate electronically excited region; CI: conical intersection; hgs: hot ground state. Black solid arrows indicate the processes of interest in this work with $\tau$ and $\tau^{\prime}$ the decay times of these processes.

system to the $\mathrm{C}_{2} \mathrm{H}_{4}^{*}$ upper state, that is a superposition of the $\pi \pi^{*}$ valence and $\pi 3 \mathrm{~s}$ Rydberg states. Since the population of the latter is small, i.e., about $10 \%$ of the valence state [22], its evolution will be disregarded here. The valence state undergoes $\mathrm{CC}$ bond twist and stretch. The absorption of a second $5^{\text {th }}$ harmonic photon from the molecule that resides in these two states produces exclusively ethylene ions $\left(\mathrm{C}_{2} \mathrm{H}_{4}^{+}\right)$. Near a $\mathrm{CC}$ torsion angle of $\varphi \approx 90^{\circ}$, an intermediate electronically excited region (IEER) of the parent molecule is reached, where multidimensional dynamics occur [23]. Since the absorption of a second photon in the IEER produces the total photofragmentation of the ethylene molecule, i.e., only the fragment ions $\mathrm{C}_{2} \mathrm{H}_{3}^{+}$and $\mathrm{C}_{2} \mathrm{H}_{2}^{+}$are obtained, this intermediate region is assumed to be much lower in energy $(2 \mathrm{eV})$ [24]. From this state, the molecule reaches a conical intersection (CI) with the ground state. This CI produces hot ground state ethylene and ethylidene $\left(\mathrm{CH}_{3} \mathrm{CH}\right.$ structure) products undergoing picosecond dynamics [22]. In the present work, we will concentrate exclusively on the ultrafast processes of the excited states indicated by the black solid arrows (see Fig. 1). $\tau$ refers to the residence time of the molecule in the Franck-Condon region for the production of $\mathrm{C}_{2} \mathrm{H}_{4}^{+}$and $\tau^{\prime}$ is the time that the molecule needs to reach the CI through the IEER. Both processes take place on the femtosecond time scale.

In the case of oxygen, the absorption of one $5^{\text {th }}$ harmonic photon brings the ground state population to the repulsive wall of the $\mathrm{B}^{3} \Sigma_{u}^{-}$electronic state, from where the subsequent dissociation occurs [25]. During dissociation, the molecule can eventually absorb a second photon and ionize [20].

The experimental setup (see Fig. 2) consists of two separate chambers, i.e., XUV production and detection chambers, separated by differential pumping. For the production of XUV harmonics, a Titanium-Sapphire femtosecond laser system operating at $10 \mathrm{~Hz}$ repetition rate, and having a carrier wavelength of $800 \mathrm{~nm}$ was used. An annular laser beam of $2 \mathrm{~cm}$ outer diameter and $15 \mathrm{~mJ} /$ pulse energy, was focused by a $3.5 \mathrm{~m}$ focal lens onto a pulsed xenon gas jet where odd harmonics were generated. For the extinction of any residual infrared radiation, a silicon wafer at Brewster's angle of $72^{\circ}$ of the fundamental radiation reflected the harmonics into the interaction region. For the selection of the $5^{\text {th }}$ harmonic a $5 \mathrm{~mm}$ thick magnesium fluoride $\left(\mathrm{MgF}_{2}\right)$ plate was used. Due to the dispersion of the material, it was necessary to precompensate the IR radiation for obtaining a nearly Fourier transform limited $5^{\text {th }}$ harmonic laser pulse. It is worth noticing that any possible residual unwanted radiation is temporarily separated from the $5^{\text {th }}$ harmonic due to the very different group velocities of the different harmonics in the $\mathrm{MgF}_{2}$ plate. Thus, the $\mathrm{MgF}_{2}$ plate was acting not only as an intensity, but also as a temporal filter. Consequently, no cross processes between the harmonics contributed to the ultrafast processes under study [18]. For the time resolved two vacuum ultraviolet photon ionization we have used a wavefront splitting arrangement. It consists of a gold spherical mirror of $5 \mathrm{~cm}$ focal length. The positioning of one of these halves is controlled by a piezo-crystal translation unit with a minimum step of $1.5 \mathrm{~nm}$. The two parts of the bisected VUV pulse were brought together into a common focus in a mixture of ethylene and krypton, or oxygen and krypton gas jet. The produced ions were detected by a time-offlight (TOF) ion-mass-spectrometer.

Figure 3 shows the two-VUV-photon ionization signal of krypton and ethylene obtained simultaneously as a function of the delay between the two VUV pulses. Figure 3(a) shows the second order AC trace for krypton. Krypton ionizes through two-photon non-resonant absorption of the $5^{\text {th }}$ harmonic. Assuming a Gaussian pulse intensity distribution, the pulse duration of the $5^{\text {th }}$ harmonic $\tau_{\mathrm{p}}=31 \pm 2 \mathrm{fs}$ could be easily extracted. Figure $3(\mathrm{~b}, \mathrm{c}$, and $\mathrm{d}$ ) shows the resonant two photon ionization of ethylene and its fragments. The measured traces were modified by the characteristic decay times of the molecule. These decay times could be deconvoluted from the measured traces once the pulse duration was determined.

The determination of the pulse duration of the $5^{\text {th }}$ harmonic and the ultrashort molecular decay times are based on the simulation of the spatial and temporal distribution of the harmonic electric field focused in the second gas jet. The spatial profile is obtained from the Debye integral [26], assuming that the split mirror is lit by an incident Gaussian beam of $6 \mathrm{~mm}$ diameter (for more details see [18]). The calculation is performed over a rectangular parallelepiped volume of width $20 w_{0}$ and length $26 z_{R}$, centered on the focal spot of the fixed part of the split mirror, with $w_{0}$ and $z_{\mathrm{R}}$ being the beam waist and the Rayleigh distance of the $5^{\text {th }}$ harmonic, respectively. For each spatial coordinate, the temporal field envelope is described by a Gaussian function corresponding to the 


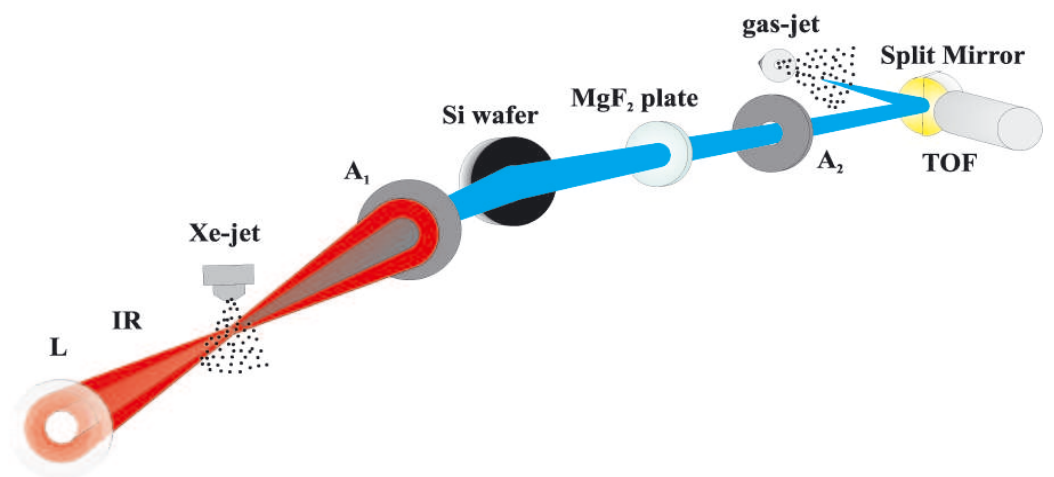

FIG. 2: Experimental setup. L: lens; $\mathrm{A}_{1}$ : aperture, differential pumping; $\mathrm{A}_{2}$ : aperture. The pulsed nozzle of the gas jet also serves as repeller of the TOF spectrometer.
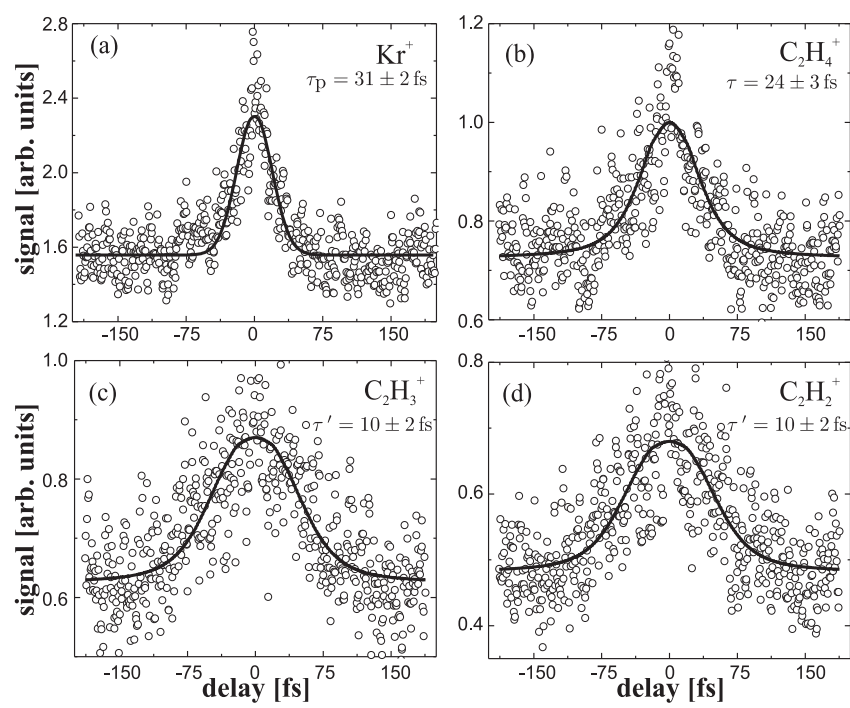

FIG. 3: Time resolved two-photon ionization trace of krypton and ethylene. Solid lines show numerical simulations: (a) $\tau_{\mathrm{p}}=31 \pm 2 \mathrm{fs}$, (b) $\tau_{\mathrm{p}}=31 \pm 2 \mathrm{fs}, \tau=24 \pm 3 \mathrm{fs}$, (c) and (d) $\tau_{\mathrm{p}}=31 \pm 2 \mathrm{fs}, \tau=24 \pm 3 \mathrm{fs}$, and $\tau^{\prime}=10 \pm 2 \mathrm{fs}$ (see text). The data are normalized to the ethylene ion signal.

pulse duration $\tau_{\mathrm{p}}$. Neither spatial nor temporal chirp of the incident XUV pulse is considered in the model.

For the krypton data presented in Fig. 3(a), the fourth power of the harmonic field modulus is integrated over the volume defined previously, and the time response of the ion detector. This is repeated for different positions of the split mirror, i.e., different time delays. The result is then averaged over each optical cycle of the VUV pulse. The resulting distribution should have a peak to background ratio close to $2[18,27]$. In the present experiments, we observed for $\mathrm{C}_{2} \mathrm{H}_{4}$ and $\mathrm{O}_{2}$ a ratio of 1.4 and 1.7 respectively. The discrepancy can be ascribed to the incident beam energy not equally shared between the two halves of the spherical mirror, and mainly to the non perfect overlap of the laser foci in the experiment. Finally, the pulse width is adjusted to provide the best agreement with the experimental data. The pulse dura- tion, $\tau_{\mathrm{p}}=31 \pm 2$ fs, inferred from the krypton AC trace, is consistent with the expected value from low order perturbation theory, and fully agrees with the value from a Gaussian distribution fit.

The parent and fragments ionic signals (see Fig. 3(b, c, and $\mathrm{d})$ ), are obtained by integrating spatially and temporally (see discussion above) the Liouville equation for a three-level system $[21,23]$ in the case of ethylene, and for a two level system in the case of oxygen [20]. For each delay, the populations of the different levels are calculated at the different space coordinates, using the field amplitude given by the Debye integral. The signal of the single photon ionization of the excited state is obtained in the Golden Rule approximation. The data presented are proportional to the cycle average of the ionization probability. In the model, for both molecules, we have neglected the depletion of the populations by the field (perturbative regime).

Figure 3(b) shows that the decay time of the parent ion, i.e., the characteristic time for the flow of population out of the Franck-Condon region, (see Fig. 1), is $\tau=24 \pm 3$ fs. The ionic fragments, since both originate from the photofragmentation of the molecule within the path leading to the CI through the IEER, have identical decay times $\tau^{\prime}=10 \pm 2$ fs (see Fig. 3(b, c)). The obtained results are in concordance with previous investigations [21-23]. The error bars for $\tau$ and $\tau^{\prime}$ are estimated considering the uncertainty in the determination of the pulse duration $\tau_{p}$ and its propagation through the model calculations to the correlated parameters $\tau$ and $\tau^{\prime}$. It is worth mentioning that in contrast to other works that relied on long wavelength single photon ionization, the use of VUV photons allowed us to track the decay of the excited wavepacket to lower electronic energies. That can explain why the decay time $\tau^{\prime}$ is observable in our work, while it was not accessible in the investigations of Farmanara [20] and Mestdagh [23].

Figure 4 shows the two-VUV-photon ionization signal of krypton and oxygen obtained simultaneously as a function of the delay between the two VUV pulses. As in the case of ethylene, we use the AC trace of krypton in or- 


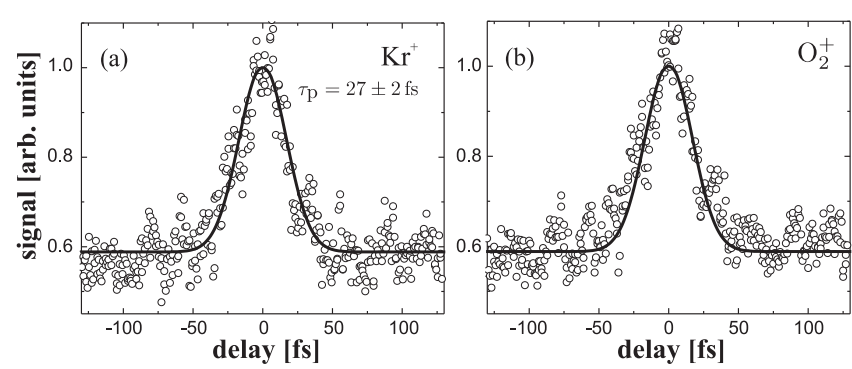

FIG. 4: Time resolved two-photon ionization trace of krypton and oxygen. Solid lines show numerical simulations: (a) $\tau_{\mathrm{p}}=$ $27 \pm 2$ fs.

der to determine the pulse duration of the $5^{\text {th }}$ harmonic. The measured value was $\tau_{\mathrm{p}}=27 \pm 2 \mathrm{fs}$. The measured oxygen trace does not differ significantly from the one of krypton. This result differs from previous investigations that have shown a relaxation time due to dissociation of $\tau_{\mathrm{O}_{2}}=40 \pm 20 \mathrm{fs}$ [20]. As a possible source of this discrepancy, we should notice that pump-probe techniques in general do not reflect the lifetime of the excited state, but rather the time that the excited wavepacket requires to leave the ionization detection window. This window depends on the final continuum state as well. Thus, different ionization wavelengths may in principle lead to different decay times. This can explain the different result between Ref.[20] and our work, since our probe wavelength $(160 \mathrm{~nm})$ is much shorter than the one used in Ref.[20] (258 nm).

In conclusion, an application of the time resolved two vacuum ultraviolet photon ionization technique in inves- tigating ultrafast molecular dynamics and the determination of molecular decay times in the temporal scale of few tens of fs has been demonstrated. This new technique is now feasible as a consequence of the high VUV/XUV intensities provided by higher order harmonic generation in gases driven by many-cycle, high-peak-power, IR, fs laser radiation. Furthermore the challenge of VUV/XUVpump-VUV/XUV-probe experiments rely simply on a modified version of the present technique that replaces the single harmonic used by two different harmonics. The demonstrated technique and methodology has a large potential that it is still to be exploited in particular utilizing shorter VUV/XUV pulses. Such pulses can be produced utilizing the recently developed polarization gating technique of many cycle $[13,28]$, high peak power pulses, or exploiting the upcoming generations of few cycle, $100 \mathrm{TW}$ laser systems, significantly increasing the temporal resolution of the method and thus challenging the few fs or sub-fs temporal regime. Finally, we should also emphasize the potentiality of the technique in applications exploiting the intense XUV light from XFEL sources [29].

The authors thank K. Kosma for providing their not yet published manuscript, and especially B.W. Shore for the most valuable discussions. This work is supported in part by the European Community's Human Potential Programme under contract MRTN-CT-2003-505138 (XTRA), MTKD-CT-2004-517145 (X-HOMES), the Ultraviolet Laser Facility (ULF) operating at FORTH-IESL (contract no. HPRI-CT-2001-00139), the ELI research infrastructure preparatory phase program (contract GA no. 212105/SP4 capacities), and the FASQUAST program (contract GA no. 214962).
[1] P. Agostini, and L.F. DiMauro Rep. Prog. Phys. 67 813855, 2004.

[2] P. B. Corkum, and F. Krausz Nat. Phys. 3, 381-387, (2007).

[3] A Scrinzi et al J. Phys. B 39, No 1, R1-R37, 2006.

[4] M.F. Kling, and M.J.J. Vrakking Annu. Rev. Phys. Chem. 59, 46392, 2008

[5] A. Baltuška et al Nature 421, 611-615, 2003.

[6] A.L. Cavalieri et al Nature 449, 1029, 2007.

[7] M. Drescher et al Nature 419, 803, 2002.

[8] M. Uiberacker et al Nature 446, 627, 2007.

[9] P. Tzallas et al Nature 426, 267-271, 2003.

[10] E.P. Benis et al Phys. Rev. A 74, 051402(R), 2006.

[11] E.P. Benis et al New J. Phys. 8, 92, 2006.

[12] Y. Nabekawa et al Phys. Rev. Lett., 96, 083901, 2006.

[13] P. Tzallas et al Nature Phys. 3, 846, 2007.

[14] D. Charalambidis et al New J. Phys., 10, 25018, 2008.

[15] T. Baeva et al Phys. Rev. E 74, 065401(R), 2006.

[16] A. Peralta Conde et al Eur. Phys. J. D. 51, 289-294
(2009)

[17] L.A.A. Nikolopoulos et al Phys. Rev. Lett. 94, 113905, 2005.

[18] O. Faucher et al Appl. Phys. B, DOI 10.1007/s00340009-3559-z.

[19] P. Farmanara et al Chem. Phys. Lett., 228, 518, 1998.

[20] P. Farmanara et al J. Chem. Phys., 111, 6264, 1999.

[21] V. Stert et al Chem. Phys. Lett., 388, 144, 2004.

[22] K. Kosma et al J. Phys. Chem. A, 112, 7514, 2008.

[23] J.M. Mestdagh et al J. Chem. Phys. 113, 237, 2000.

[24] P.D. Foo, and K.K. Innes J. Chem. Phys. 60, 4582-9, 1974.

[25] J.L. Lin et al J. Chem. Phys. 109, 1758, 1998.

[26] M. Born and E. Wolf Principles of Optics (Pergamon, New York, 1968).

[27] P. Tzallas et al J. Mod. Opt., 52, 2, 321, 2005.

[28] D. Oron et al Phys. Rev. A 72, 063816, 2005.

[29] R. Mitzner et al Opt. Expr. 16, 19909, 2008. 